\title{
Echinococosis lung: A Cyto-Histopathogical Study in Two Cases
}

\author{
Rimi Pandey ${ }^{1 *}$, Nivedita Yadav ${ }^{1}$, Padam Kumari Agarwal ${ }^{1}$, Suruchi Shukla ${ }^{2,}$ Mustafa Ali 2 , and Pooja Singh ${ }^{1}$ \\ ${ }^{1}$ Pathology department. Vivekananda Polyclinic \& Institute of Medical Sciences. Lucknow, India \\ ${ }^{2}$ Microbiology Department. Vivekananda Polyclinic \& Institute of Medical Sciences. Lucknow, India
}

\begin{abstract}
Echinococcosis is a parasitic infestation caused by the cestode of the genus Echinococcus. Man serves as an intermediate host and becomes infected by accidental ingestion of eggs from soil, water or by direct contact with the definitive host (dog). The present communication deals with two cases of echinococcosis of the lung. The presenting chief complaints were of breathlessness and dry cough. Routine haematological investigations revealed relative lymphocytosis in both the cases. However X-ray chest and CT showed a mass in the left lung in one case and a mass in the right lung in another case. For further evaluation CT guided fine needle aspiration cytology (FNAC) was performed in case one and the final diagnosis of hydatid cyst lung was established. In another case surgery was performed. Histopathological examination, established the diagnosis of echinococcosis lung. Both the patients were treated accordingly and they responded well to antihelminthic regime of therapy.
\end{abstract}

Keywords: Echinococcosis Lung, Lung Mass, Cytology, Hydatid cyst,

\section{Introduction}

Hydatidosis also known as Echinococcosis remains a serious health hazard in endemic countries, like India. It is a zoonotic disease where the parasite completes its lifecycle involving definitive host (dogs). The man is infested by accidental ingestion of larval forms of Echinococcus. In the present cases, there was no history of contact with any of the animals, responsible for transfer of the infection to human beings. In humans, hydatid disease commonly involves the liver $(70 \%)$ and the lungs $(25 \%)^{[1]}$. In the later case, the children are more commonly infected as compared to adults. In the present communication, both the cases were young boys. Presumptive diagnosis of hydatid cyst was suspected on imaging techniques, however cytopathological or histological confirmation was considered essential prior to therapy.

FNAC may lead to rupture of the cysts, resulting in hypersensitive/anaphylactic reaction of mild to moderate degree and also no reaction at all ${ }^{[2,3]}$. But, this procedure is still being carried out routinely, at some centres to confirm the diagnosis ${ }^{[3]}$, since FNAC is an economical and simple technique and well accepted procedure. In the present cases, one of them was finally diagnosed on FNAC alone, whereas surgery was performed in another case, followed by histopathological confirmation.

\section{Case Reports}

Case 1: A 14-years-boy attended the outpatient department of a tertiary care hospital with chief complaints of loose stools, breathlessness and dry cough since last 3 months.
Routine investigations were within normal limits except for relative lymphocytosis.

X-ray chest showed a mass near the pericardium. It was confirmed on CT scan, which revealed a massive lesion in left side thorax lying in relation to the pericardium showing homogeneous enhancement and specks of calcification, probably neoplastic in nature. CT guided FNAC was performed to confirm the diagnosis. Multiple slides were prepared, some of them were wet fixed in 95\% alcohol for Pap's and H:E stain and others were kept air dried for MGG stain. All the slides were studied and reported by cytopathologist.

On microscopic examination the cytosmears were covered by necrotic debris and showed neutrophilic and lymphocytic infiltrate. Reactive squamous epithelial cells (figla), multinucleated giant cells (figlb) and proliferated capillaries (fig1c\&d) were present. On careful screening occasional refractive lanceolate hooklets (figle \& f) were present embedded in the necrotic debris. In another slides irregular thin fragments of bluish enucleated membranous structures covered by inflammatory cells (figlg) were present. The surface was lined by thin germinal type of cells (Fig 1h), but laminations were not clear in cytosmears.

To rule out the possibility of any associated infection, special stains for microorganisms were carried out, such as for tuberculosis, the cytosmears were stained with ZiehlNeelson's stain, which was negative for acid fast bacilli. But the spines were seen more prominently as AFB 
negative structures (fig1g). Also the Grocott's stain was performed to rule out any associated fungal infection. No fungal elements were present, but spines were better seen in the background of inflammatory exudate (fig $1 \mathrm{~h}$ ). In one of the smear one scolex (fig1i) was also seen. Thus based on these findings final diagnosis of hydatid cyst (echinococcosis) lung was given. Later on, serology and repeated stool examination was advised which came out as negative.

The patient was given a course of anti-helmithic antibiotics (Albendazole, Metrogyl and Ceconorm). He responded well as observed after two months of treatment. His repeat chest X-ray revealed only pneumonitis. No mass was seen.

Case 2: An 8- year- old boy presented to the hospital with chief complaints of dry cough and respiratory distress since 2 days. Cough increased on sitting position and relieved on lying down. A year back he had a history of chest trauma following a fall after which he developed hematemesis. On examination of the chest, there were decreased breathing movements on the right side of chest and decreased breath sounds on the same area.

He did not respond to conventional medication and started having frequent episodes of cough and respiratory distress. He was referred to this tertiary care centre for further management.
His chest $\mathrm{x}$-ray revealed a cystic lesion in right lung (fig.2a). HRCT chest showed a large well defined cystic space, occupying lesion in the right lung along the interlobar fissure (fig.2b). A differential diagnosis of encysted pleural effusion or a hydatid cyst was made. Hematological investigation revealed mild leukocytosis only.

Thoracoscopic excision of the cyst along with adjoining lung tissue from right middle lobe of lung was performed. Both the specimens were submitted for histopathological examination for confirmation of diagnosis. Post operatively antibiotics were started. He tolerated the anti-helmithic drugs well. Post-op x-ray revealed air in the cyst. The child did well after the operation and had no fresh complaints.

Histopathological Examination: An opened up thin white glistening cyst measuring 9x3x1 cm. was received. (fig.2c). Another piece of adjoining lung tissue measured $2 \times 0.6 \times 0.5$ $\mathrm{cm}$. in size. Both the specimens were thoroughly examined and multiple pieces were submitted for microscopic examination. Histopathological examination revealed that the cyst wall was composed of laminated acellular structure, lined by germinal epithelium. Tissue from the lung revealed congested alveoli occupied by thin pinkish bits of hyadatid cyst wall and multiple scolexes of hydatid (fig.2e\&f).

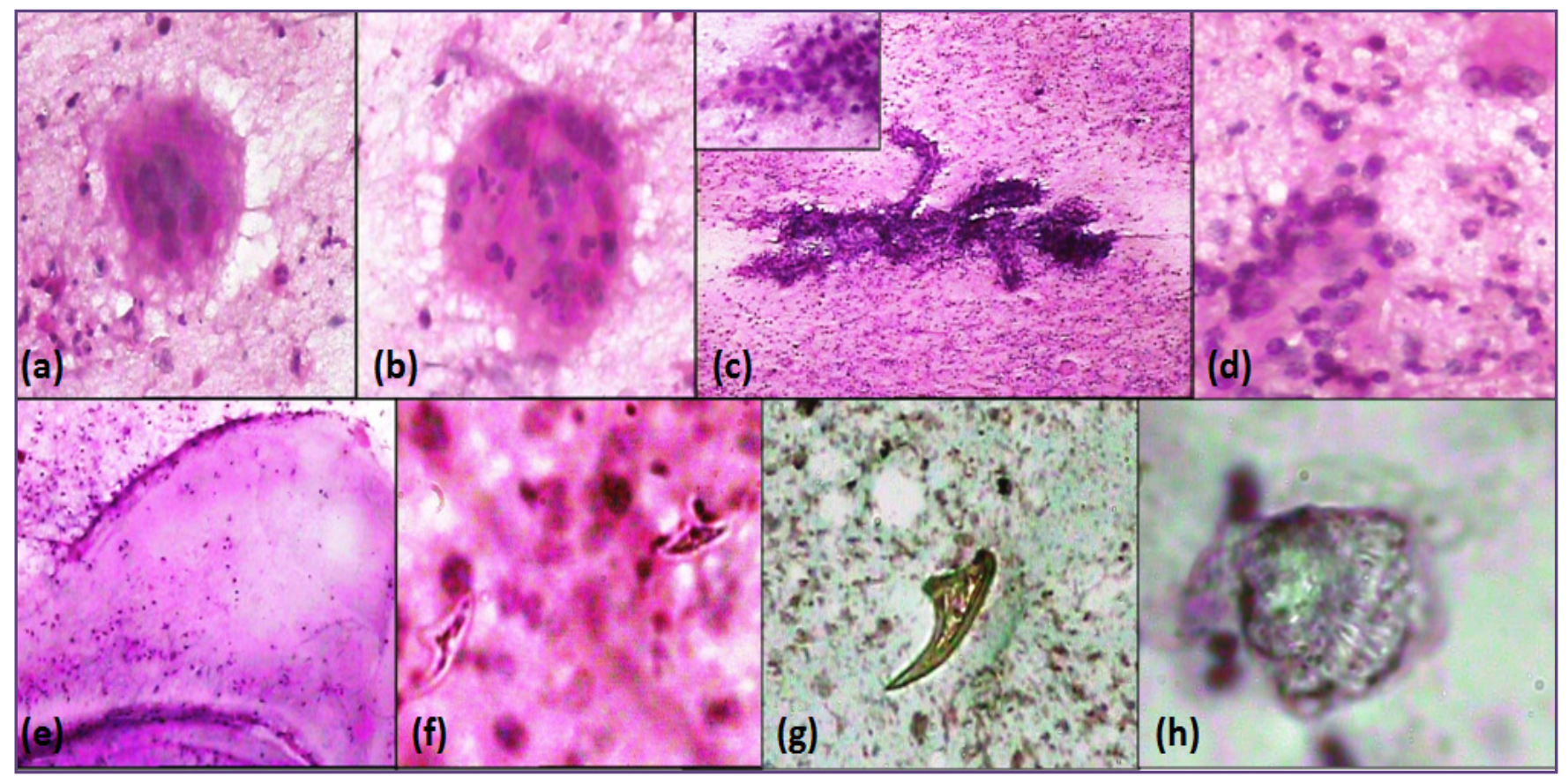

Fig. 1: (a-g) Cytosmears: (a) Multi-nucleated foreign body type of giant cells (H: E X 40). (b) Multinucleate giant cells with phagocytic leucocytes (H: E X 40). (c) Proliferated capillaries (H: E X 10), Higher magnification (inset, H: E X 100). (d) Necrotic background with bi-nucleate macrophage and a capillary (H: E X 40). (e) Membranous structures and necrotic debris (MGG $X$ 10). (f) Hooklet lying on the necrotic background (H: E X 40). (g) Hooklets (Grocott X 100). (h) Scolex (MGG X 100). 


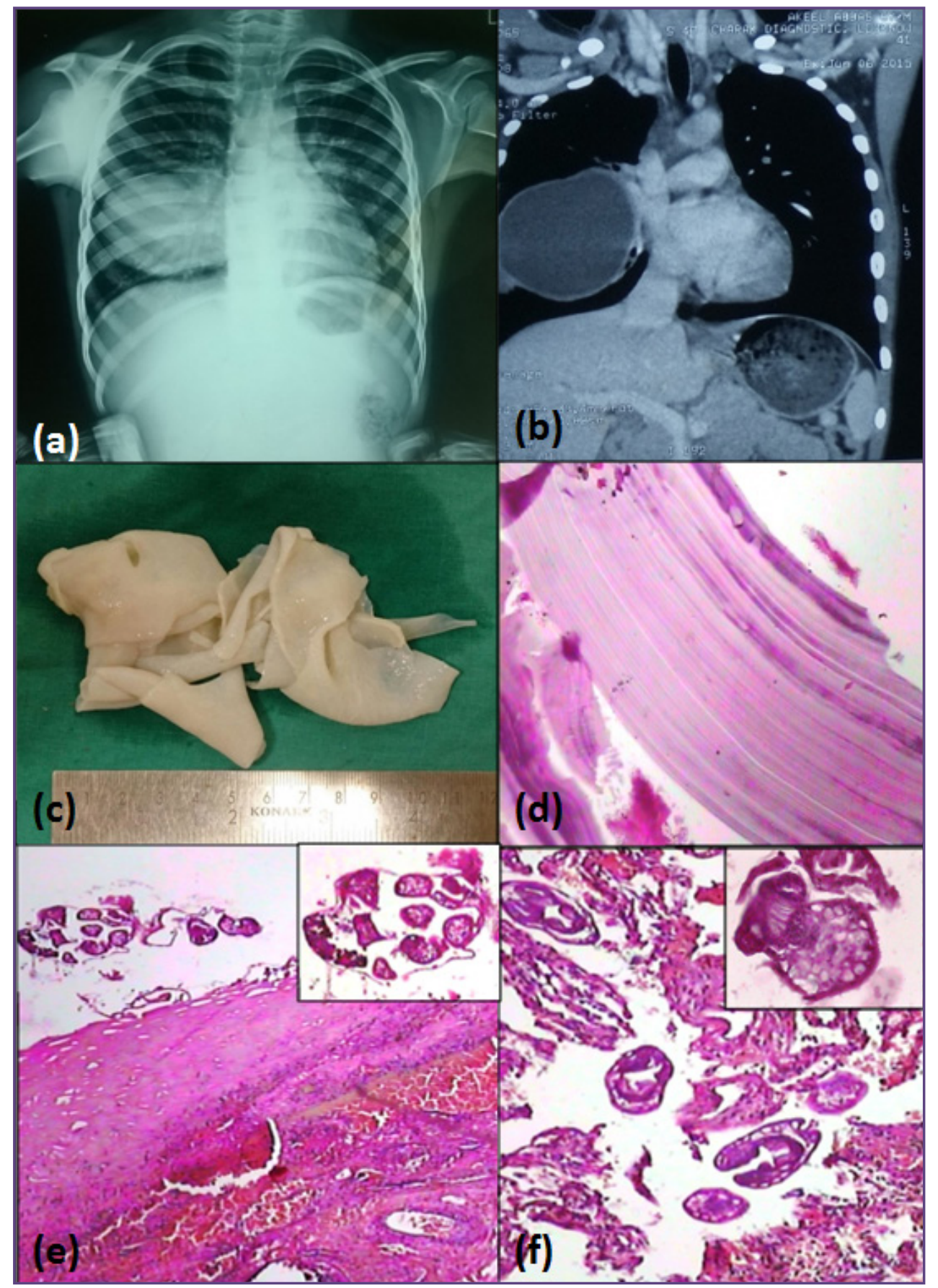

Fig. 2(a-f):(a) Chest x-ray showing cystic lesion in right lung (b) HRCT chest showing large well defined cystic space along the inter-lobar fissure (c) Gross specimen: Opened up cyst (d)Histopathology: Laminated acellular cyst wall lined by germinal layer (H: E X 40). (e) Multiple scolex along the congested bronchial wall (H: E X 10); Multiple scolex (inset, H: E X 40). (f) Congested alveoli occupied by multiple scolex of hydatid wall (H: E X 10); scolex (inset, H: E X 100).

\section{Discussion}

Hydatid cyst is an infectious disease which is most commonly caused by the eestode Echinococcus granulosus and rarely by Echinococcus multilocularis ${ }^{[1]}$. In lung hydatidosis one lobe, usually the basal one of the right lung is involved in $72 \%$ of the cases. ${ }^{[3]}$ Our case no. 1 had a mass along the pericardium in the left lung, while the case no. 2 had cyst in the middle lobe of the, right lung which are not 
common sites. The disease in the lung is common in the first two decades of life. Incidentally, both the present cases were young boys.

The signs and symptoms depend on the size and the site of the lesion. The common presenting symptoms are cough, chest pain, and breathlessness. The case no.1, presented with chest pain and breathlessness while the other presented with chest pain and respiratory distress following chest trauma. He was asymptomatic before the trauma, but his chest symptoms were aggravated after trauma to the chest wall. Skouti et.al in 2015 reported a similar case of hydatid cyst following chest trauma. ${ }^{[4]}$

Routine investigations in both the cases revealed leucocytosis with a normal eosinophil count. Haematological and biochemical tests are not reliable indicators to establish the diagnosis of hydatid disease. Casoni's test and repeated stool examination were negative in the present cases.

Radiological studies e.g. Plain X-ray films, Ultrasonography, Computed Tomography (CT), and Magnetic Resonance Imaging (MRI) are of great value in diagnosing and detecting the Echinococcal cysts and also determining the anatomic extent of the disease.

Histological/cytological confirmation is mandatory before the treatment, especially surgery ${ }^{[6]}$ Cytological findings have complementary role in the management of patients, particularly those with negative serology and normal eosinophil count. The finding of protoscolices, hooklets or hydatid membranes on cytosmears are the hallmarks of the diagnosis of an Echinococcus cyst. ${ }^{[7,8]}$ In our first case, FNAC confirmed the diagnosis of Echinococcus and the patient was treated accordingly.

Another important feature in the cytosmears in our case no.1 was extensive tissue reaction, the knowledge of these changes is essential to avoid false positive diagnosis of a neoplasia or hamartoma. Possibility of various differential diagnoses like fungal infection, granulomatous lesions etc. was also ruled out by studying the smears for microbiological infections on special staining for fungus and acid fast bacilli. Anaphylaxis, which is a recognized complication, was not observed in the present case, as reported in earlier studies ${ }^{[8,9]}$.The patient was given a course of anti-helminthic drugs to which he responded well and his symptoms almost disappeared on follow up after about a year. The second child is doing well after surgery. No complications were noted in the case even after two years follow up.

A word of caution, that the Hyadatidosis should be taken into account in paediatric patients of both the sexes, especially the boys presenting with pulmonary symptoms, irrespective of the side and the involved lobe of the lung by a unilateral pulmonary cystic/mass lesion. After radiological suspicion of a mass or an encysted pleural effusion in any lobe, a guided FNAC is mandatory to confirm the diagnosis before instituting medical/ surgical treatment for the proper treatment and better prognosis of the patients.

\section{References}

1. Eckert J, Schantz P, Gasser R,et al. Geographic distribution and prevalence. In: Eckert J, Gemmell MA, Meslin FX, Pawlowski ZS, eds. WHO/OIE Manual on Echinococcosis in Humans and Animals: a Public Health Problem of Global Concern. Paris: World Organization for Animal Health, 2001: 100-141.

2. Eckert J, Deplazes P. Biological, epidemiological, and clinical aspects of echinococcosis, a zoonosis of increasing concern. ClinMicrobiol Rev. 2004; 17: 107-135

3. Ahmad S, Jalil S, Saleem Y, et al. Hydatid cysts at unusual sites: reports of two cases in the neck and breast. J Pak Med Assoc 2010; 60: 232-4.

4. Sokouti M, Shokouhi B, Sokouti M et al. Giant Pulmonary Hydatid Cyst and Trauma in a 9 Year-Old Child: A Case Report. Open Respir Med J. 2015; 9: 67-69.

5. Basavana GH, Siddesh G, Jayaraj BS, et al. A ruptured hydatid cyst of the lung. JAPI.2007; 55:141-45.

6. Tekinbas C, Turedi S, Gunduz A, Erol M. Hydatid cyst disease of the lung as an unusual cause of massive hemoptysis: a case report. Journal of Medical Case Reports $.2009 ; 3: 21$.

7. Mitra S, Kundu S, Das S, Mukherjee S. Mediastinal hydatid disease: an unusual presentation. Indian J Chest Dis Allied Sci.2010; 52: 245-47.

8. Das DK, Bhambhani S, Pant CS. Ultrasound guided fine needle aspiration cytology: diagnosis of hydatid disease of the abdomen and thorax. Diagn Cytopathol 1995 March; 12 (2): 173-6.

9. Agarwal P K, Husain N, Singh B N. Cytologic findings in aspiratedhydatid fluid. Acta Cytol 1989; 33: 652-654.

*Corresponding author:

Dr Rimi Pandey, M-8, sector-i, Near Prabhat Chauraha, Jankipuram, Lucknow (226021) India

Phone: +91 9554464552

Email: rimi.may26@gmail.com

Financial or other Competing Interests: None.

Date of Submission : 26.11.2016

Date of Acceptance : 02.04.2017

Date of Publication : 29.05.2017 